\title{
Modelling the Impact of Ground Planes on Aircraft Transmission Cable Impedance
}

\author{
I.T. Kiely, P.J. Norman, B.G. Stewart \\ University of Strathclyde \\ 16 Richmond St, G1 1XQ, Glasgow
}

\begin{abstract}
With the recent movement in the aircraft industry to have a more electric based secondary power system, new challenges are being uncovered, particularly with the electrical wiring interconnect system. These systems and their insulation are expected to be exposed to significantly higher voltage and frequency stresses. Complicating matters, aircraft power transmission cables are often unshielded, yet are located in close proximity to a ground plane due to the aircraft metallic structure. Electromagnetic interactions between the two in this environment are poorly understood, particularly with respect to how the cable impedance changes for higher frequency signals. Using finite element analysis (FEA), this paper investigates how field stress conditions for high frequency components change as the cableground distance changes. A wider discussion of the impact of the mapped behavior on future aircraft electrical wiring design and airframe integration will also be presented. Findings demonstrate that despite cable-ground plane distance being within the standard limits, the cable characteristics can still significantly change, with a $3 \mathrm{~cm}$ distance leading to a $15 \%$ change in impedance.
\end{abstract}

\section{INTRODUCTION}

There has been growing momentum in the aircraft industry to move pneumatic and mechanical based loads to the existing electrical power network, and is described in literature as the more-electric-aircraft (MEA) concept [1]. As a result, the electrical capacity is significantly higher and more diversified, necessitating a greater presence of converters on the network. However, the higher power density of these converters demands the use of higher frequency switching, and consequently, higher frequency emissions potentially being injected into the electrical wiring interconnect system (EWIS). In the commercial aircraft sector, electromagnetic interference (EMI) standards are defined in DO-160G [2]. Common mode conducted electromagnetic interference limits from converters are defined between $150 \mathrm{kHz}$ to $152 \mathrm{MHz}$, with lower frequency EMI being created from pulse width modulation (PWM) harmonics, and higher frequency EMI from switching transitions [3]. Military standard MIL-STD-146G defines acceptable EMI to the range of $10 \mathrm{kHz}$ to $10 \mathrm{MHz}$ from all power leads, including earth returns [4]. Complicating matters further, parts of the EWIS are unshielded for the purpose of weight reduction. This has significant implications as electromagnetic interference between the unshielded cable and the metallic structure of the aircraft itself can occur. This EMI subsequently alters the cable characteristics, leading to changes in the cable impedance and hence the current carrying characteristics of the conductor. Of particular importance is how the cable impedance changes with respect to frequency and with the cable distance from the aircraft metallic structure, as together these determine how susceptible the EWIS is to high frequency transient currents. Given the anticipated increase of converters on board, and hence associated increased risk of high frequency faults and transients occurring, it is paramount that the EWIS behavior is understood.

This paper investigates the impact of having unshielded cables in close proximity to a grounded structure, and the implications that this can have on high frequency current flow. The paper will conclude with a discussion on how these unknown cable parameters can have lasting implications on future aircraft network design, as well as mitigation strategies that could be employed.

\section{CABLE CHARACTERISTIC AND AIRCRAFT CONSIDERATIONS}

It has been well established that as the frequency in a cable increases, the resistance also increases, in a phenomena known as the skin effect [5]. Primary current flow in the conductor creates an internal magnetic field, which in turn induces internal eddy currents. These eddy currents are induced in such a way that the eddies closer to the center of the cable oppose the current flow, while eddies near the surface of the conductor flow with the primary current. The result of this is that as frequency increases, the current density predominantly flows near the surface of the conductor, thus for sufficiently high frequencies, this can have a notable impact on the resistance and internal inductance of the cable.

With the use of unshielded cables on aircraft, it is also imperative to take into account the external magnetic field produced by the conductor and how it interacts with the surroundings. This proximity effect, is similar to the skin effect, where induced eddy currents between the cable and metallic body surroundings result in higher concentrations of current density on the conductor area closest to the cable and the ground plane, resulting in an increase in cable resistance and cable external inductance [6].

This work has been supported through the UK Engineering and Physical Sciences Research Council (EPSRC) Centre for Doctorial Training in Future Power Networks and Smart Grids, and with Rolls-Royce 


\section{AnAlytical SOLUtions To CABLE-GROUND PROBlem}

\section{A. Per-Unit-Length Resistance}

Skin depth, $\delta$, is defined as the distance below the conductor surface where the current density has fallen to $1 / e(\sim 36 \%)$ of the current density at the surface of the conductor, and underpins why the cable per-unit-length parameters are not constant for high frequencies [7]:

$$
\delta=\frac{1}{\sqrt{\pi \mu_{0} \mu_{r} \sigma f}}
$$

where $\delta$ is skin depth (m), $\mu_{0}$ is permeability of free space, $\mu_{r}$ is permeability of air, $\sigma$ is electrical conductivity $(\mathrm{S} / \mathrm{m})$, and $f$ is frequency $(\mathrm{Hz})$.

The skin depth is typically observed to impact per-unit-length parameters when it is greater than twice the conductor radius, $r_{c}$, i.e., when $r_{c}<2 \delta$ [8]. The per-unit-length resistance, $r_{i 0}(\mathrm{~m})$, for skin depths below this threshold is:

$$
r_{i o}=\frac{1}{\pi \sigma r_{c}^{2}}
$$

For skin depths greater than this, the per-unit-length resistance is a function of $1 / \sqrt{f}$, as shown in equation (3):

$$
r_{i o}=\frac{1}{\pi \sigma\left(r_{c}^{2}-\left(r_{c}-\delta\right)^{2}\right)}
$$

\section{B. Per-Unit-Length Inductance}

The inductance of a cable can be separated into two components, inner and outer inductance, created from the flux permeating inside and outside the conductor respectively.

\section{1) Internal Inductance}

For low frequencies, the assumption is made that the current density is equal through the core. Hence, the internal inductance, $l_{\text {int }}(\mathrm{H})$ can be approximated as:

$$
l_{\text {int }}=\frac{\mu_{0} \mu_{r}}{8 \pi} .
$$

This value is approximately $50 \mathrm{nH}$, and is the maximum internal inductance of a $1 \mathrm{~m}$ length, isolated cable. However, for high frequencies the per-unit-length internal inductance must be expressed as a function of frequency due to the skin effect [9]:

$$
l_{\text {int }}=\left(\frac{1}{2 \pi r_{c}}\right)\left(\sqrt{\frac{\mu_{0} \mu_{r}}{4 \pi f \sigma}}\right) .
$$

\section{2) External Inductance}

The flux linkage between the cable and the earth plane defines the per-unit-length external inductance $l_{\text {ext }}(\mathrm{H})$, hence the distance of the ground plane, $h(\mathrm{~m})$, is important. As there is no frequency dependency, this external impedance defines the minimum inductance of the cable over a frequency response, and is typically significantly greater than the maximum internal inductance [10]:

$$
l_{\text {ext }}=\left(\frac{\mu_{0} \mu_{r}}{2 \pi}\right)\left(\ln \left(\frac{h}{r_{c}}+\sqrt{\left(\frac{h}{r_{c}}\right)^{2}-1}\right)\right)
$$

However, both the internal and external inductance equations only hold for when the cable to ground plane distance is significantly larger than the conductor radius, $h \gg r_{c}$. At small cable-ground distances, the proximity effect interaction between both elements leads to the current density no longer being equally distributed around the radius of the conductor and assumptions about the internal and external field no longer being valid.

\section{Per-Unit-Length Capacitance}

By method of images the per-unit-length capacitive, $c_{i 0}(\mathrm{~F})$, coupling between the cable and ground plane is as follows:

$$
c_{i 0}=\frac{2 \pi \varepsilon_{0} \varepsilon_{r}}{\cosh ^{-1}\left(\frac{h}{r_{c}}\right)},
$$

where $\epsilon_{0}$ is the permittivity of free space and $\epsilon_{r}$ is the relative permittivity of the medium [8].

\section{FEA OF CABLE-GROUND SYSTEM}

In this study, a $1 \mathrm{~m}$ length, 540 strand, $14.8 \mathrm{~mm}$ finished cable diameter of power feeder aeronautical cable Nexans SP 799 [11], set to 10 A current flow, was simulated situated above a copper ground plane of $1 \mathrm{~mm}$ depth, with this ground plane emulating the aircraft metallic structure. The cable insulation was modeled as a single $1.25 \mathrm{~mm}$ thick layer of PTFE, with a relative permittivity of 2.1 and relative permeability 1 . It is possible to use FEA to evaluate per-unit-length cable parameters. The FEA software "Quickfield" was used for this analysis [12]. It has been shown in past studies that a stranded cable design for FEA more accurately models the conductor, due to the need to also model the skin and proximity effect per strand [13]. The change in magnetic field strength over a range of frequencies can be observed in Figures 1 and 2 respectively.

In Figure 1, the magnetic field that can be seen emanating from the stranded conductor is stronger at the side closest to the ground place. Figure 2 shows how this magnetic field changes with frequency, with the inner magnetic field at high frequencies being reduced to negligible levels. The presence of the ground plane leads to a $4-9 \%$ difference in the peak magnetic field strength between the top and bottom of the conductor, depending on the frequency. The skin effect can also be observed here, with a sharp drop off in the internal magnetic field at frequencies above $10 \mathrm{kHz}$.

Different geometric scenarios comprising of $1 \mathrm{~cm}$ increments in the cable-ground distance were studied, along with an adjacent and no ground scenario, with a cable current of $10 \mathrm{~A}$. 


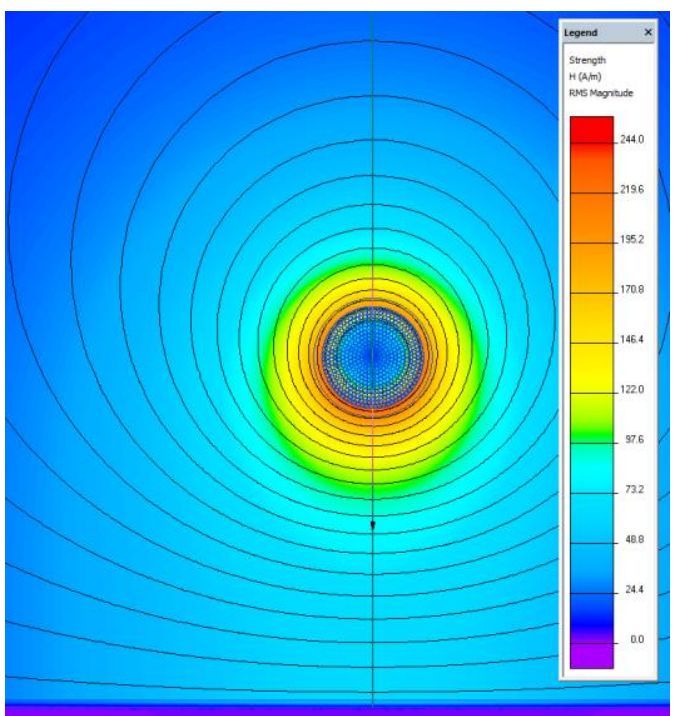

Fig. 1. Magnetic field emanating from the conductor, $\mathrm{f}=1 \mathrm{kHz}$, cable-ground distance $=5 \mathrm{~cm}$.

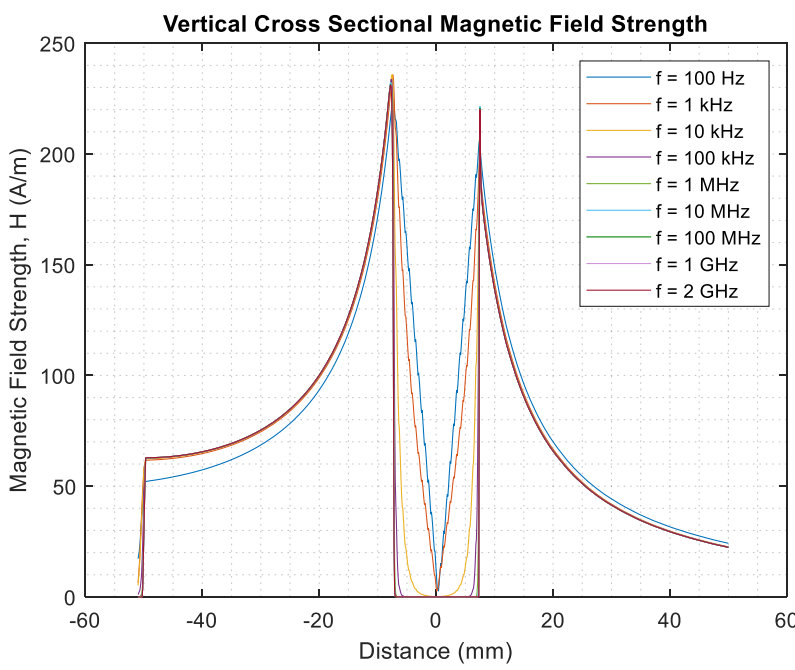

Fig. 2. Magnetic field strength for set frequencies across the $5 \mathrm{~cm}$ cableground scenario. The centre of the conductor is at $0 \mathrm{~mm}$, and ground plane at $50 \mathrm{~mm}$.

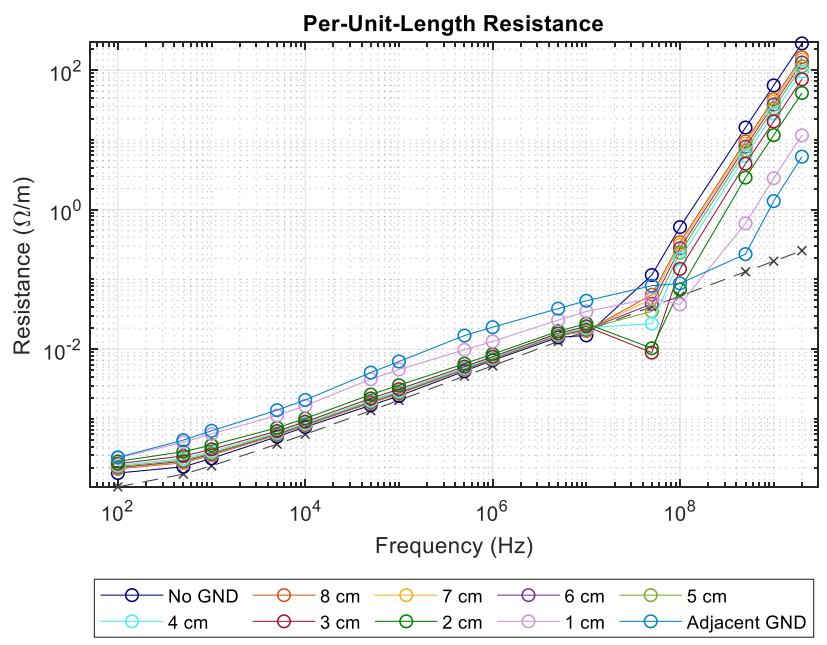

Fig. 3. FEA result of per-unit-length resistance against frequency.

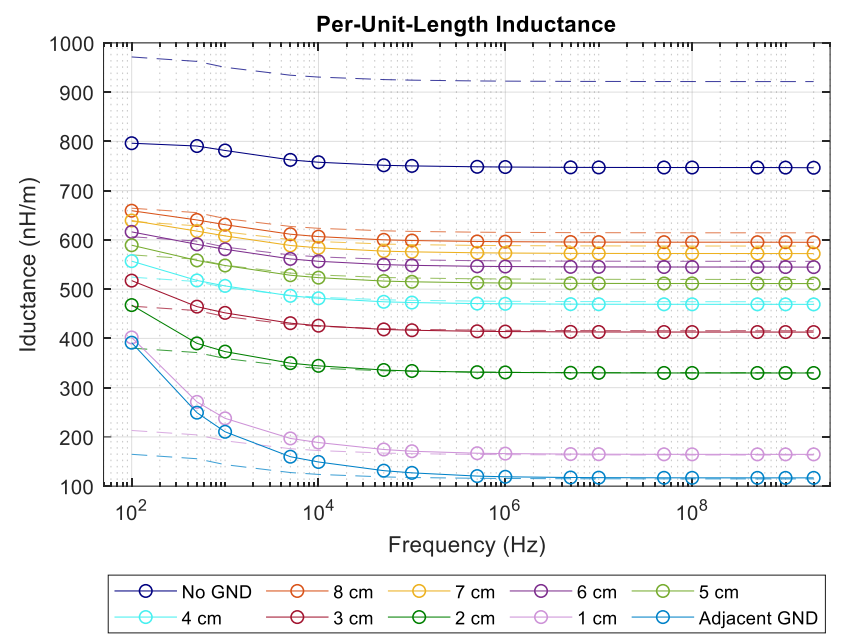

Fig. 4. FEA result of per-unit-length inductance against frequency.

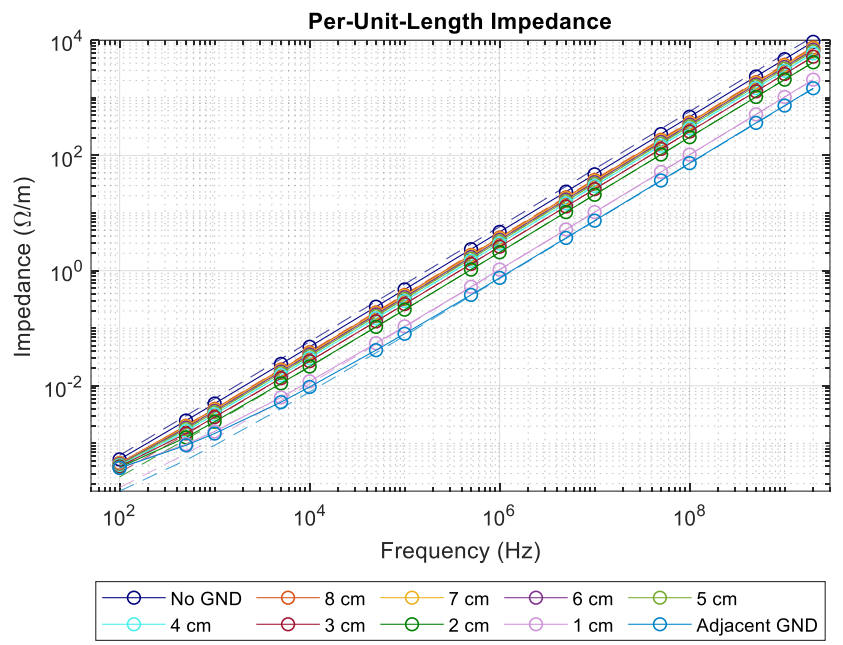

Fig. 5. FEA result of per-unit-length impedance against frequency.

A frequency sweep from $100 \mathrm{~Hz}-2 \mathrm{GHz}$ was then performed for each scenario, and the per-unit-length values for resistance, inductance, and impedance found using the Time-HarmonicMagnetic (THM) method. Results are shown in Figures 3-5 respectively for per-unit-length resistance, inductance and impedance and are compared to the analytically defined values denoted by dashed lines of the same color.

Due to the THM method being unable to calculate the capacitance, the reactance is purely a function of inductance. At frequencies greater than $5 \mathrm{kHz}$ the reactance begins to dominate over the resistance. Thus, the cable-ground distance has a large influence on the magnitude of the impedance. There is a large divergence (up to a factor of 6.4 between no ground and adjacent ground scenarios) between impedances for cableground distances that can be observed. For the resistance, the impact of the proximity effect of the ground plane on the cable can be observed with an offset, which has not been taken into account in the analytical model. While FEA is a close approximation of the analytical calculations, at high frequencies, approximately above $50 \mathrm{MHz}$ where the skin depth is extremely small, the resistance varies significantly. Similarly, the effects of this can also be seen in the inductance. For small 
cable-ground distances, at low frequencies the inner inductance vastly exceeds the theoretical maximum of $50 \mathrm{nH}$. Both of these can be explained by the extremely small phenomina being modelled (skin depth and proximity effect respectively), thus requiring significantly finer meshing to suffiently model the cable.

The per-unit-length capacitance of the cable can be calculated with electrostatic simulations, where a voltage of $115 \mathrm{~V}$ is placed across the cable. As an electrostatic simulation only considers a constant fixed voltage, the per-unit-length capacitance frequency response is impossible to derive, however the cable-ground capacitance with respect to cableground distance can still be found. Results are shown in Figure 6 , with the cable-ground capacitance increasing exponentially as ground plane distance decreases. FEA analysis provides a similar cable-ground capacitance to the analytically determined value, at a mean percentage difference of $1.23 \%$. However, for extremely small distances the simulated capacitance is larger than expected. This can again be explained through the dielectric insulation material of the cable being a higher proportion of the medium compared to the air between the cable and the ground, and hence a larger cable-ground capacitance.

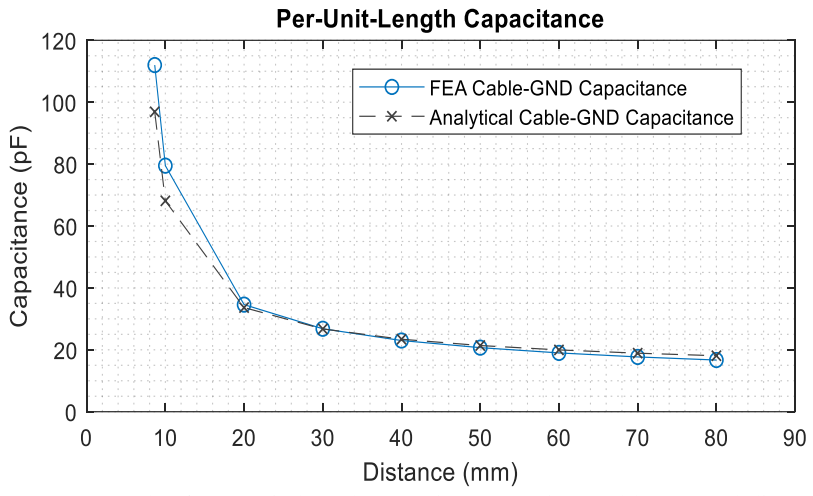

Fig 6. FEA result of per-unit-length capacitance against cable-ground distance.

\section{DISCUSSION}

The DO-160G electromagnetic disturbance standards state that the cable-ground plane distance must be kept to a minimum of $5 \mathrm{~cm}$. However, for distances above this specification, there are still large variations in the impedance (the average analytical percentage difference between $8 \mathrm{~cm}$ and $5 \mathrm{~cm}$ cableground distance being $15.12 \%$ ). This percentage difference is relatively consistent, even at lower frequencies. These results may have large repercussions on the future design of the EWIS. As aircraft are steadily becoming more electrified, the quantity and rating of cables are also progressively increasing, hence increasing consequences of EMI and adding greater uncertainty to the per-unit-length characteristics of cable bundles. Of particular importance is the susceptibility of the EWIS to high frequency transients, as the cable impedance at these high frequencies is largely influenced by the inductance over the cable. With the secondary subsystem of the aircraft having a greater dependency on the EWIS, this has potential to evolve into greater problems in the future. Further investigation needs to be made on how to strike a balance between sufficient cable shielding from EMI and the weight investment that would be required. Another avenue of research is how to directly measure individual cable bundle characteristics, which would expedite high quality installation and maintenance regimes. Finally, construction of higher model fidelity and faster computation FEA of the EWIS and its interaction with the aircraft metallic body can also aid the development and understanding of cable characteristics and the expected behavior of the aircraft electrical grid. A more effective understanding of the influence of increased cable insulation on capacitance evaluations would also assist in understanding how high frequency signals may propagate along cable runs.

\section{CONCLUSIONS}

This paper shows how the presence of a ground plane can largely influence cable per-unit-length characteristics, how analytical theory matches to FEA simulations, and the further implications these results have on the EWIS. Results have been presented that demonstrate how existing standards for unshielded cable in an aircraft are not sufficient for consistent characterization of cables in future applications. Future work will explore how this uncertainty in impedance affects the EWIS for high frequency transients.

\section{REFERENCES}

[1] X. Roboam, "New trends and challenges of electrical networks embedded in "more electrical aircraft," in 2011 IEEE International Symposium on Industrial Electronics, 2011, pp. 26-31.

[2] IEEE GlobalSpec, "RTCA DO-160 Environmental Conditions and Test Procedures for Airborne Equipment,” 2019. [Online]. Available: https://standards.globalspec.com/std/9894152/rtca-do-160.

[Accessed: 15-May-2019].

[3] A. Charalambous, X. Yuan, and N. McNeill, "High-Frequency EMI Attenuation at Source with the Auxiliary Commutated Pole Inverter," IEEE Trans. Power Electron., vol. 33, no. 7, pp. 5660-5676, 2018.

[4] US DoD Interface Standard, "MIL-STD-461G: REQUIREMENTS FOR THE CONTROL OF ELECTROMAGNETIC INTERFERENCE CHARACTERISTICS OF SUBSYSTEMS AND EQUIPMENT."

[5] G. S. Smith, "A simple derivation for the skin effect in a round wire," Eur. J. Phys., vol. 35, no. 2, 2014.

[6] M. Xie, H. Wang, X. Zhang, Z. Gao, and W. Li, "Study on impedance characteristic of aircraft cables," Int. J. Aerosp. Eng., vol. 2016, pp. $1-11,2016$.

[7] M. Raven, "Skin effect in the time and frequency domain comparison of power series and Bessel function solutions.," J. Phys. Commun., 2018.

[8] P. R. Clayton, Analysis of Multiconductor Transmission Lines, 2nd Editio. John Wiley \& Sons, 2007.

[9] M. S. Raven, "Experimental measurements of the skin effect and internal inductance at low frequencies," Acta Tech., vol. 60, no. 1, pp. 51-69, 2015.

[10] P. R. Clayton, Inductance: Loop and Partial. John Wiley \& Sons, 2010.

[11] Nexans, "SP 799 Datasheet," 2019. [Online]. Available: http://www.nexans.fr/eservice/France-en/pdf-

family_25396/SP_799.pdf. [Accessed: 15-May-2019].

[12] Tera Analysis Ltd, "Quickfield," 2019. [Online]. Available: https://quickfield.com/. [Accessed: 15-May-2019].

[13] V. Dos Santos, N. Roux, B. Revol, B. Sareni, B. Cougo, and J. Carayon, "Unshielded Cable Modeling for Conducted Emissions Issues in Electrical Power Drive Systems," in Proc. of the 2017 International Symposium on Electromagnetic Compatibility - EMC EUROPE, 2017, vol. 3, pp. 6-11. 